\title{
Audit
}

\section{Referrals for panic disorder to a clinical psycho- pharmacology research unit}

\author{
Luiz Dratcu, Honorary Senior Registrar and Alyson Bond, Honorary Principal \\ Clinical Psychologist, The Institute of Psychiatry and Maudsley Hospital, \\ Denmark Hill, London SE5 8AZ
}

Clinical research attempts to find out the best way to treat patients and audit attempts to make sure that patients are treated in the best possible way. The two are thus inextricably linked and should benefit from each other (Smith, 1992). In running a clinical research unit in which the personnel involved (two psychiatrists and two psychologists) have only honorary contracts, it is sometimes difficult to match the service offered to the clinical population required. A constant flow of patients does not mean a constant flow of those prepared or suitable to participate in research projects. Unlike clinical referrals, which are received passively, referrals of patients for research have to be looked for, even when treatment is on offer.

The auditing of health services involved in clinical research could provide data useful not only for the service, but also on practical matters related to the organisation of research activities and sometimes on the research topic itself, thus offering material which could be helpful to other units and professionals also working in clinical research.

\section{The study}

The Clinical Psychopharmacology Unit is part of the Department of Psychiatry at the Institute of Psychiatry. Clinical activities are carried out under the Bethlem Royal Hospital and Maudsley Hospital special health authority. The unit is basically concerned with research. Many studies are done with healthy volunteers but some involve patients. However, the unit also performs clinical duties unrelated to research. Patients referred to the unit come from different sources and for different reasons. They may be tertiary referrals from other psychiatrists seeking a second opinion or GP referrals for specialist assessment and treatment. Research poses different practical problems and recruiting patients to take part in clinical research studies is a challenging task.

The study examined different sources of referral to the unit over one year and which of these patients participated in an experimental study of panic disorder (PD). Referrals of patients with PD, a condition for which a prevalence of over $2 \%$ in the population has been reported (Wittchen et al, 1991) have been sought from a variety of sources.

(a) Routine referrals: for assessment and/or treatment to the department who did not take part in any research.

(b) Requested referrals: various referral sources were contacted and asked to refer patients in need of treatment.

(c) Clinical volunteers: an informative article on panic disorder was written for a popular magazine and sufferers willing to take part in a research programme were invited to write to the unit.

\section{Findings}

Routine referrals: 62 clinical patients were seen in the unit during a 12-month period; $24 \%$ of these involved a single appointment, $71 \%$ were seen for follow-up appointments in the out-patients department and $5 \%$ required in-patient treatment. In all, $70-80 \%$ of patients were discharged or referred on within the period. This excludes patients who either did not attend or who agreed to participate in a research project and were subsequently seen in the clinic. None of them suffered from PD or could be included in the study.

Requested referrals: letters sent to $70 \mathrm{GPs}$ in the hospital catchment area, to all Maudsley Hospital consultants and to all consultants in psychological medicine at Kings College Hospital ( $\mathrm{KCH}$ ) were highly ineffective. Two referrals came from GPs; one did not attend and one did not want to participate. Only personal contact with the sources on a regular basis resulted in some success. Two patients from within the department completed the study. Regular visits to the Emergency Clinic of the Maudsley 
Hospital resulted in three referrals: one did not attend, one had to be excluded and one dropped out. Weekly visits to the Department of Psychological Medicine of $\mathrm{KCH}$ out-patients clinic where GP letters were screened resulted in 15 referrals: five did not attend, three did not want to participate, two had another diagnosis, one dropped out and four completed the study. However, 22 referrals after one year of strenuous work could hardly be considered a success; even less so when only a quarter referred (6) completed the study, less than the number (7) who failed to attend their first appointment.

Clinical volunteers: 60 letters were received from readers of the published article. The letters were scrutinised and questionnaires were sent to 39 who claimed to suffer from PD after reading the article. Respondents were directly asked if they were prepared to take medication as part of a research programme. Of the PD sufferers, $31(80 \%)$ returned the questionnaire and $27(70 \%)$ indicated that they would be prepared to take part. Appointments were sent to 20 of the respondents: five did not attend, four did not want to participate, one had to be excluded, one had a different diagnosis and one dropped out. This left eight ( $40 \%)$ who completed the study.

\section{Comment}

A clinical research unit such as ours has the structure to deal with clinical and research patients. These may be interchangeable to some extent but in this study represented two different populations. This should be considered whenever a new project in clinical research is devised as a considerable amount of time and effort is expended in both initially obtaining the participants and then providing clinical follow-up.

It will always be difficult to get patients to take part in research and it is important to take account of the various necessary stages. Suitable referrals may not be compliant or may have to be excluded. A large number do not attend the first appointment. Some are afraid of being labelled "psychiatric" patients or of drug treatment. However, drop-outs can be decreased by giving full information, stressing the benefits and providing freely available telephone support.

Sources of clinical patients are not always the best when psychopharmacological research is involved. In this case, a service was offered and advertised to GPs for a relatively common condition which is impairing and has a high comorbidity with other disorders (Wittchen et al, 1991) but this resulted in very few referrals. There could be many reasons for this: GPs do not read routine letters; GPs do not recognise/diagnose $P D ; P D$ patients do not consult their GP (Wilkinson, 1989). Psychiatric colleagues were also contacted and did not respond, so there may be few PD patients in the psychiatric services.

However, PD is indeed present in the community as shown by the response to one article in the press. Many patients realised for the first time that they had a disorder which was treatable only after reading the article. Others had previously sought help from medical services without success. An additional important finding of this study is that the press can be a useful ally in educating the public and informing them of current research and we may need their help to de-stigmatise psychiatry and mental illness so that patients seek help through more usual channels.

\section{References}

SMITH, R. (1992) Audit and research. British Medical Journal, 305, 905-906.

Wilkinson, G. (1989) Referrals from general practitioners to psychiatrists and paramedical mental health professionals. British Journal of Psychiatry, 154, 72-76.

WitTChen, H., Essau, C. A. \& KRIEG, J. Anxiety disorders: similarities and differences of comorbidity in treated and untreated groups. British Journal of Psychiatry, 159 (Suppl. 12), 23-33.

Questionnaire on panic disorder available from Dr Dratcu on request. 